\title{
Clustering in mental health payment by results: a critical summary for the clinician $^{\dagger}$
}

\section{David Yeomans}

\begin{abstract}
SUMMARY
Mental health payment by results $(\mathrm{PbR})$ is a disruptive new prospective payment system intended to replace National Health Service block contracts in England and provide a mechanism for opening up the mental health economy. Patients are allocated to one of 21 treatment clusters, each with a different price or tariff. Clinicians perform cluster allocation using the Mental Health Clustering Tool. The clustering process makes demands on clinicians' time even with support from information systems. Clustering is novel and it is unclear how it will work in practice. The process is likely to be susceptible to gaming.
\end{abstract}

\section{LEARNING OBJECTIVES}

- Understand that the clinical process of diagnostic classification is different from the financial process of clustering categorisation.

- Understand the importance of learning the clustering tool ratings definitions in order to make accurate cluster allocations.

- Recognise that mental health payment by results is driving the widespread adoption of outcomes measurement.

\section{DECLARATION OF INTEREST}

None.

'Public services are never better performed than when their reward comes in consequence of their being performed, and is proportioned to the diligence employed in performing them.'

Adam Smith, The Wealth of Nations (1776)

Mental health payment by results ( $\mathrm{PbR}$ ) is precisely the sort of mechanism that Adam Smith recommended almost 340 years ago to fund public services. It is a financial device that can be used or misused, depending on your orientation, in a variety of ways that this article will explore. A companion article in Advances by Rowena Jacobs describes PbR systems in detail (Jacobs 2014).

The purpose of mental health $\mathrm{PbR}$ is to effect change. Currently, psychiatric services in England are paid for with block contracts that do not vary according to activity. Adam Smith recommended that public services are reimbursed in proportion to the volume of activity performed and this is what mental health PbR aims to do.

\section{Clustering and outcome measures}

Despite the name, mental health PbR does not yet pay on the basis of results or outcomes, but by activity. Not all activity is of the same nature or degree, so the authors of mental health $\mathrm{PbR}$ have developed a currency to address case mix. Thus, more demanding provision is funded at a higher level than less complex or intense work. This case-mix approach is called clustering. It is the equivalent of healthcare resource groups in other systems.

Mental health clusters are assigned using the Mental Health Clustering Tool (MHCT), a structured rating of behaviour, symptoms, risk, functional status and history. When clinicians assign a cluster to a patient they are allocating a fixed price for that patient's care for a set period of time called a cluster review period. Any qualified provider of mental health services can bid to offer a service to a patient at that fixed price. Providers that spend less on service provision than the cluster tariff will make a profit, and those spending more will lose money. The system therefore encourages providers to work within the price limit of each cluster, and it is hoped that this will drive down the costs of service provision. Of course, there have to be checks and balances to encourage quality as well as efficiency. In future, various forms of outcome measure will be used to help determine quality.

\section{The administrative burden of clustering}

Clinicians may be diverted somewhat from direct patient care by clustering, which is a process equivalent to raising invoices in a retrospective payment system. This diversion may increase as demands for quality measures and outcomes are included. Computerised administrative systems could offer support to minimise loss of
David Yeomans is a consultant in adult psychiatry with Leeds and York Partnership NHS Foundation Trust. He is interested in the ethical use of information systems to demonstrate meaningful outcomes. Correspondence Dr David Yeomans, Hawthorn House, St Mary's Hospital, Green Hill Road, Leeds LS12 30E, UK. Email: david. yeomans@nhs.net

${ }^{\dagger}$ For a commentary on this article, see pp. 235-236, this issue. 
productivity, and staff will need to adapt to the new environment. In acute services, $\mathrm{PbR}$ is supported by administrative staff called clinical coders. Clinicians record diagnoses and procedures and then the coders transform these into tariff codes. This separation of clinicians from accounting means that they are unlikely to be drawn into gaming the system (see the section on Gaming below), a protection that mental health $\mathrm{PbR}$ does not afford. In mental health $\mathrm{PbR}$, the clinicians are also the coders.

\section{Diagnosis and clustering processes are not the same}

Clustering will make all professional groups in mental healthcare adopt a new classification system to support the flow of money around the National Health Service (NHS). Doctors in particular need to understand that this new financial classification system differs from diagnostic systems. Clusters borrow heavily from the clinical language of medical diagnosis, but they have very different meanings. When a practitioner makes a clinical judgement that someone has a first-episode psychosis this is because the person reports a syndrome of psychotic symptoms such as hallucinations and delusions, and is experiencing distress and disability. When the same practitioner allocates the same person to a mental health cluster called first-episode psychosis they do so in order to receive payment of $£ 10606$ during that cluster's review period of 12 months. Clustering is a financial process wrapped up in clinical terms.

The Royal College of Psychiatrists (2014) does not support the current clustering model. The College position statement on mental health

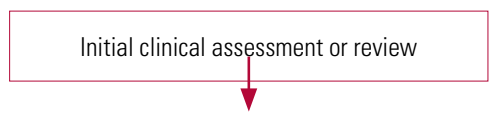

Seek supervision if needed

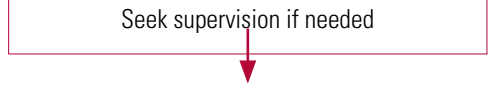

Complete an MHCT on your IT system, using the guidance in the MHCB on HoNOS ratings, historical ratings and super class

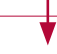

Apply the clustering algorithm on your IT system or refer to the MHCB to decide which cluster to allocate

Review clustering when the cluster review period is up or the patient experiences a significant change in status. Repeat the MHCT. Use the care transition protocols in the MHCB to see whether criteria for a step-up or discharge are met. If not, check whether a step-down or continuance of the current cluster is appropriate

Flow chart for clustering. HoNOS, Health of the Nation Outcome Scales; MHCB, Mental Health Clustering Booklet; MHCT, Mental Health Clustering Tool. payment systems expresses dissatisfaction that diagnosis is a secondary consideration to the clustering system. College members are concerned that 'the implementation of the current system would risk severe destabilisation, both financially and organisationally'. Perhaps the absence of diagnosis challenges the position of psychiatrists in the system.

Making a diagnosis is a process of using information gained from conversations and examinations to categorise people into groups. It is a fundamental part of the medical model. In psychiatry there is little pathological evidence to inform diagnoses, so the profession has developed diagnostic standards, based on clinical assessments, published as international diagnostic manuals. The system of allocating clusters is a different categorisation process, with 21 clusters instead of several hundred diagnoses. Clustering does not use diagnosis because diagnosis is not well correlated with mental healthcare expenditure other factors, such as marital status and electoral ward of residence, are better predictors of cost (Oyebode 2007).

The MHCT relies in large part on a set of scales developed by the Royal College of Psychiatrists' Research Unit in the 1990s called the Health of the Nation Outcome Scales (HoNOS) (Wing 1996), so it does have clinical credentials, but there is no scheme to translate backwards and forwards between diagnosis and clustering. A person with a diagnosis of first-episode psychosis could be allocated to one of several clusters, such as 'psychotic crisis', 'first-episode psychosis' or 'ongoing or recurrent psychosis (high symptom and disability)'. A person with a cluster allocation of 'psychotic crisis' may have a clinical diagnosis of schizophrenia, mania, depression or personality disorder. The cluster is a single price that covers all psychiatric comorbidities in a single category. A person with multiple psychiatric diagnoses (e.g. depression, substance misuse and personality disorder) will only receive one mental health cluster.

\section{How to do clustering}

The cluster is derived from ratings of scales $2-13$ of the HoNOS and five additional historical ratings. The first HoNOS scale (overactive, aggressive, disruptive or agitated behaviour (current)), is not used. Together, these items comprise the MHCT, details of which are laid out in the Mental Health Clustering Booklet (Department of Health 2013a). A flow chart for clustering is shown in Fig. 1. It is generally expected that professionals will use the cluster scoring system described in the Mental Health Clustering Booklet. The booklet is a thick 
manual that needs to be reproduced in colour to allow practitioners to make complex decisions using a set of definitions, flow charts and tables. It is now supported by a computerised clustering algorithm. The algorithm allows the HoNOS and historical ratings to be converted into a small number of suggested clusters, each with a calculated probability. This can be tried out as an Excel spreadsheet (Department of Health 2013b). The algorithm is a significant practical step forwards for experienced clinicians, who can now complete an initial MHCT and allocate a cluster in less than 10 minutes. Less experienced staff may need more time, including time for supervision.

Clustering can be carried out by an individual practitioner or a team. The team approach could help generate higher-quality cluster assignments since it provides immediate peer supervision.

Once the clinical assessment or review is complete, practitioners will log into their IT system, select the patient record and open the computerised MHCT. They then fill in the ratings in line with their clinical knowledge of the patient. They also decide whether the person being clustered has a non-psychotic, a psychotic or an organic disorder. These three domains are called 'super classes'. They make a big difference to the final cluster allocation. The Mental Health Clustering Booklet can be used to guide the final cluster allocation, but running the clustering algorithm will be faster and simpler. Both require a decision about how to order the ratings in the clusters. To do this strictly, the practitioner using the booklet must ensure that the essential ratings for each cluster (red ratings) are considered first. The algorithm user must switch on the 'red rules'. Further information can be found in the Mental Health Clustering Booklet.

The clustering booklet gives explicit descriptions of rating scales that are generally precise and understandable, but staff may misunderstand the ratings for several items because the item title, read independently of the description, may be misleading. Box 1 gives some examples.

The clinician can choose whatever cluster they deem fit, but it is hard to see why anyone would disagree with the clustering algorithm without a good reason, since clustering defines itself, and to diverge from the algorithm could introduce random variation. It is important for doctors and non-medics not to transfer diagnostic assumptions into the clustering process by confusing clinical terms with the cluster names. At the time of writing, the algorithm is only beginning to be implemented on IT systems in some services: 18 months after it was first published in December 2012. Use of the variance cluster (0) when no
BOX 1 Common rating errors with the mental health clustering tool

Here are some examples of item titles which, read independently of the item descriptions, may be misleading.
Item 12: Problems with occupation and activities (current)

Read the description carefully: 'Rate the overall level of problems with quality of day-time environment. Is there help to cope with disabilities, and opportunities for maintaining or improving occupational and recreational skills and activities? Consider factors such as stigma, lack of qualified staff, access to supportive facilities, for example staffing and equipment of day centres, workshops, social clubs, etc. Do not rate the level of functional disability itself: this should be rated at Scale 10.' (It may help to think of item 12 as 'Problems with accessing occupation and activities'.)
Item B: Repeat self-harm (historical) The description includes the advice: 'Do not include harm with intention of killing self (rated at Scale 2)'. This is confusing since scale 2 is about current risk of non-accidental self-injury, including suicide (current risk is defined as being within the past 2 weeks). Item E: Vulnerability (historical) is more appropriate. This item asks staff to: 'Rate failure of an individual to protect themselves from risk of harm to their health and safety or well-being'. So past suicide attempts are rated in item $E$, not item $B$. Item D: Engagement (historical) Note that this scale asks staff to rate both under-engagement and over-engagement (dependency). cluster decision can be made will be discouraged since it is not likely to be well funded.

\section{Cluster review}

After a period of time specified within each cluster definition (from 4 to 52 weeks; Table 1), or sooner if clinical status changes significantly, there should be a review of the cluster allocated. The patient's cluster allocation may stay the same, or step up or step down. A step up represents an increase in a combination of factors, including severity of needs, acuity of symptoms and complexity of care. A step-down cluster should reflect a sustained reduction in severity, acuity, complexity or intensity of care.

The IT system should remind clinicians when reclustering is necessary so that they can repeat the MHCT. Then they should observe the criteria in the cluster transition protocols in the Mental Health Clustering Booklet. These are not yet available for the computerised algorithm. If criteria for a step-up cluster are met, the patient is reallocated to this cluster. If not, the practitioner should check the local discharge criteria. If these are met, then the patient may be discharged. If the discharge criteria are not met, then, using the new MHCT result, the clinician should consider whether criteria are met for either moving to a step-down cluster or remaining in the current cluster. The chosen cluster should reflect the assessed clinical status of the patient. Patients making a clear sustained recovery should step down to lower clusters and patients getting more unwell should step up to higher clusters that can 
TABLE 1 Clusters arranged in order of descending value (unit cost per day) ${ }^{a}$

\begin{tabular}{|c|c|c|c|c|}
\hline Code & Cluster label & $\begin{array}{l}\text { Maximum cluster review } \\
\text { period, weeks }\end{array}$ & Unit cost per day, $f$ & $\begin{array}{l}\text { Unit cost per maximum } \\
\text { cluster review period, } f\end{array}$ \\
\hline 14 & Psychotic crisis & 4 & 86.23 & 2415 \\
\hline 17 & Psychosis and affective disorder (difficult to engage) & 26 & 53.24 & 9690 \\
\hline 15 & Severe psychotic depression & 4 & 46.56 & 1304 \\
\hline 13 & Ongoing or recurrent psychosis (high symptom and disability) & 52 & 40.57 & 14809 \\
\hline 16 & Dual diagnosis & 26 & 35.84 & 6522 \\
\hline 10 & First episode psychosis & 52 & 29.06 & 10606 \\
\hline 12 & Ongoing or recurrent psychosis (high disability) & 52 & 26.45 & 9653 \\
\hline 8 & Non-psychotic chaotic and challenging disorders & 52 & 23.92 & 8731 \\
\hline 21 & Cognitive impairment or dementia (high physical or engagement need) & 26 & 23.58 & 4291 \\
\hline 20 & Cognitive impairment or dementia (high need) & 26 & 22.30 & 4059 \\
\hline 5 & Non-psychotic (very severe) & 26 & 21.75 & 3959 \\
\hline 6 & Non-psychotic disorders of over-valued ideas & 26 & 18.50 & 3367 \\
\hline 7 & Enduring non-psychotic disorders (high disability) & 52 & 18.16 & 6628 \\
\hline 4 & Non-psychotic (severe) & 26 & 16.69 & 3037 \\
\hline 11 & Ongoing recurrent psychosis (low symptoms) & 52 & 15.22 & 5556 \\
\hline 3 & Non-psychotic (moderate severity) & 26 & 11.41 & 2076 \\
\hline 2 & Common mental health problems (low severity with greater need) & 15 & 10.66 & 1120 \\
\hline 19 & Cognitive impairment or dementia (moderate need) & 26 & 10.19 & 1855 \\
\hline 1 & Common mental health problems (low severity) & 12 & 9.25 & 777 \\
\hline 18 & Cognitive impairment (low need) & 26 & 5.75 & 1046 \\
\hline
\end{tabular}

resource more intensive care packages. This is a very complex process, especially for general adult teams, which need to be familiar with the whole range of clusters. There could be conflicts between the cluster transition protocols, the MHCT result and the clustering algorithm, so it is helpful that the clinician is able to make the final choice of cluster reallocation.

\section{When should clustering take place?}

The Mental Health Clustering Booklet (Department of Health 2013a) recommends that clustering be undertaken in each of the following situations:

- at the end of the initial assessment (within two contacts, or 2 days for in-patients)

- at all planned care programme approach (CPA) or other formal care reviews

- at reassessments specified by the cluster review period (e.g. at least every 4 weeks for cluster 14, psychotic crisis)

- at any other point where a significant change in planned care is deemed necessary (e.g. unplanned reviews, urgent admissions).

\section{When should clustering not take place?}

Reclustering should not automatically take place at service transitions (e.g. discharge from a ward). It should happen only when a cluster reaches the end of its predetermined review period, or a patient's needs change significantly so that the current cluster is inaccurate and the care package no longer matches those needs. This is particularly important when a patient is getting better. Downgrading a cluster too soon while needs remain high will, in effect, withdraw resources for treatment. Since the clinical imperative will be to continue treatment, the effect will be a loss of income. This is a form of down-coding, or underestimating the cost of care.

In early trials of clustering, if clinicians completed clusters at the end of a hospital admission when a patient was better, rather than at the start when the patient was unwell, they would down-code the whole hospital episode to a 'common mental health problem' instead of a more appropriate cluster such as 'psychotic crisis'. In future, this basic misunderstanding will be 
disastrous for income. Clinical staff need good training and supportive IT systems to deliver accurate clustering.

Clustering requires a patient focus rather than a service focus, but many trusts are organised and managed along service lines. The National Service Framework and other drivers encouraged services to fragment care into 'functional models', which means that patients move from one functionalised service to another with considerable frequency instead of remaining with a consistent care team. Trusts with a service-line focus may decide that clustering needs to take place at every service transition. This could lead to unnecessarily frequent cluster reviews, which will divert time away from direct patient care. This also risks inadvertent premature down-coding if patients make temporary improvements and then relapse. Cluster reviews should reflect sustained changes in each patient's status and these changes will not always fit neatly with service transitions.

\section{The cost of clustering}

Clustering has direct and indirect costs due to demands on clinical time, IT system development and training. The clinical cost will be borne directly by practitioners and indirectly by patients. Further requirements are already planned, including increased data collection for the mental health minimum data-set (MHMDS), additional intervention codes and a suite of outcome measures (Department of Health 2013c), all of which may reduce time for direct patient contact.

Considerable investment may be needed to make IT systems fit for purpose, since providers could stand to lose income if clustering is not carried out reliably. Most clinicians in functionalised services will not be able to keep up with cluster transition reviews without being reminded, because teams do not have continuous relationships with patients. Consequently, IT systems will also need to support care pathways as they are introduced, especially in general mental health services. General teams do high volumes of work covering the full range of mental health PbR clusters, National Institute for Health and Care Excellence (NICE) guidance and care pathways. Care coordinators have large case-loads. It is inconceivable that the clustering work can be done efficiently without very good IT support. IT systems should also be capable of synthesising the data from the clustering process, including clinical outcomes, and delivering reports back to clinical teams. This feedback of outcomes to teams is a potential return on the investment of clinical time in the clustering process and could help clinical teams improve quality.
Guidance on implementing PbR for mental health services recommends that trusts create a PbR lead, a PbR finance lead and a PbR informatics lead, as well as $\mathrm{PbR}$ champions in specific areas (Department of Health 2013d). It also recommends that organisations have a number of clinical experts in the use of clusters who cascade their expertise to other staff. Training and supervision in mental health clustering should be led by clinicians, who have experience on the ground of using the system. The Royal College of Psychiatrists and other training bodies offer courses in mental health clustering. The training should be regularly updated to account for developments in mental health $\mathrm{PbR}$ and clustering. Training should be aimed at helping clinicians perform clustering efficiently and reliably while retaining their time for patient contact.

\section{Outcomes $^{\ddagger}$}

Commissioners are advised to consider not just the cost of provision but quality and outcomes as well (Department of Health 2013c: p.11). Clinicians will need to demonstrate good outcomes to distinguish themselves in the new market of fixed prices. The guidance for mental health $\mathrm{PbR}$ and clustering recommends that services collect three main types of outcome: clinician-rated outcome, patientreported outcome and patient-reported experience.

Clinician-rated outcome measures (CROMs) include the HoNOS element of the MHCT. It is a good measure to start with but it may be affected by gaming, since relatively inconsequential changes in ratings on a single HoNOS item can shift patients into clusters that deliver much higher or lower income. Separate CROMs may be more appropriate in future and there are plenty to choose from.

The second type of outcome is assessed using a patient-reported outcome measure (PROM). Again there are several such measures, but the PbR guidance (Department of Health 2013d) makes specific reference to the Warwick-Edinburgh Mental Well-being Scale (WEMWBS). This is a brief patient-rated questionnaire that gives a single total score. It has been validated in a general population sample involving students (Tenant 2007) and work is progressing to look at clinical populations (Department of Health 2013c: p. 12). A shorter version of the scale (SWEMWBS) has now replaced the WEMWBS as the recommended PROM for reporting purposes.

The final type of measure is a patient-reported experience measure (PREM). Here the guidance is less developed, but it will probably involve the 'friends and family' question (Department of
FFor a discussion of the use of outcome measures see Lewis G, Killaspy H (2014) Getting the measure of outcomes in clinical practice. Advances in Psychiatric Treatment, 20: 165-171. Ed. 
Health 2013c: p. 12) in which patients are asked to rate (on a five-point scale) the likelihood of them recommending the service to their friends and family.

A critical factor for success with outcomes is the use of the IT infrastructure to help prompt, collect and analyse outcomes for teams to reflect upon as well as to report them centrally. Mental health $\mathrm{PbR}$ will in effect introduce routine outcome measurement across mental health services in England for the first time.

\section{What is not covered by mental health clustering?}

Cluster prices do not include the cost of the initial assessment, rates for which need to be negotiated separately. Comorbid physical health problems treated by mental health services are not currently included within clusters. Local negotiations will need to work out how to pay for this additional care. Mental health $\mathrm{PbR}$ guidance suggests that physical comorbidities can be recorded using the MHMDS and this can be used to charge funders (Department of Health 2013c: section 18.5). Many specialist services are currently excluded from mental health clustering and these are listed in Box 2.

\section{Likely challenges with clustering}

Mental health $\mathrm{PbR}$ is a slowly developing process. To date, practitioners may have had a little training and been asked to get only one cluster recorded on each patient. In future, this will become a constant

BOX 2 Services not covered by clustering and mental health $\mathrm{PbR}$

- Non-contract (e.g. out-of-area treatment)

- Acute hospital admissions to treat mental health problems (e.g. self-harm in A\&E, or re-feeding for anorexia nervosa on a medical ward)

- Child and adolescent mental health services (CAMHS)

- Forensic and secure services

- Perinatal psychiatric services

- Tertiary eating disorders services

- Services for gender dysmorphia

- Specialist mental health services for deaf people

- Services for severe obsessive-compulsive disorder and body dysmorphia

- Severe personality disorder services

- Specialist addiction services

- Specialist psychological therapy services
- Acquired brain injury services

- Specialist autism and Asperger services

- Liaison services (but these may be included in the future)

- Tertiary services for complex and/or treatment-resistant disorders

- Intellectual disability services for nonmental health needs

- Discrete improving access to psychological therapy (IAPT) services

- Mental health services under a general practitioner contract

Notes

The relationship between mental health $\mathrm{PbR}$ and personal health budgets is evolving; $\mathrm{PbR}$ may help set personal budgets

Several pilots to widen the scope of mental health $\mathrm{PbR}$ are underway rolling routine and therefore could divert time from clinical work to finance. Clinical staff need to engage positively with this financial process and its challenges, which have no cultural precedent in the English NHS. Excellent change management skills are needed in NHS organisations to make the introduction successful.

Clustering is based on a medical model and so focuses on deficit: people are seen as broken and in need of fixing. The HoNOS, however, talk of problems and symptoms that need rectifying. The notion of strengths and recovery despite symptoms is not addressed directly by the MHCT and historical problems will cast a long shadow. A study comparing clusters with six scales of the Model of Human Occupation Screening Tool (MOHOST), used in occupational therapy, found that clustering did not represent the same needs groupings as the MOHOST, suggesting a difference between occupational needs and mental health clusters (Lee 2013). Another study found that nearly half of people with an intellectual disability attending a community team could not be clustered at all (Radhakrishnan 2012). It is likely that the clustering tool will evolve to address such shortcomings.

Some research into outcomes of treatment for psychosis indicates that good long-term outcomes are not necessarily linked to symptom reduction. Medical treatment of psychosis can reduce symptoms in the short term, something that has a financial incentive in mental health $\mathrm{PbR}$. The same incentive could worsen long-term functional outcomes because long-term medical treatment of symptoms may be associated with poorer longterm functional outcomes (Wunderink 2013).

Clusters may be too 'high level' to capture detailed variation in costs of service provision within a cluster. It is likely that providers' cluster costs will be higher for patients from deprived areas where poor housing, unemployment and substance misuse are more common. Within the same cluster, services may be cheaper to provide in affluent areas where patients have better resources and support networks. This may require a financial adjustment of some sort to encourage providers to offer services to deprived areas. This may make a national tariff scheme difficult to implement.

Sometimes clinical disagreement about MHCT ratings may cause clusters to oscillate. Different clinicians may make very different ratings, so PbR will throw a spotlight on variation in practice. Practitioners and managers should be careful not to have knee-jerk reactions to the data that mental health PbR throws up. Training and supervision can address variation. 
The main practical problem of clustering will be that of poor IT support. If the IT system cannot help practitioners do the job of clustering quickly and efficiently, then they may fail to achieve acceptable levels of compliance or quality. The solution is to build very good clustering support into IT systems that are universally used throughout the organisation. In future these systems must also support the use of outcomes and care pathways in combination with clustering.

\section{Gaming}

Financial incentives change behaviour. The behaviour that organisations and individuals engage in to maximise income is sometimes called gaming. General practitioner commissioners have complained that trusts in acute physical care game their PbR systems (Vize 2013). Gaming can create perverse activity. For example, some trusts facing financial penalties for failing to meet 4-hour waiting time targets in accident and emergency (A \& E) departments apparently made patients wait outside in ambulances so as to delay the actual entry to A $\&$ E, when the clock starts ticking (Watts 2012).

\section{Up-coding}

Financial gaming is limited with block budgets that are remote from clinicians, but mental healthcare practitioners using $\mathrm{PbR}$ will be immersed in the financial process. With $\mathrm{PbR}$, each patient has a regular financial assessment by a clinician, who assigns a price to the patient. Practitioners usually want to get the best service for their patients and may tweak their clustering tool ratings up a bit to achieve a higher-paying cluster. This will be easy to do as they become familiar with the idiosyncrasies of the clustering algorithm. As a result, clinicians might assign more patients to high-value clusters (this is called up-coding and increases income without increasing quality). It is likely that internal and external audit processes (another cost) will be needed to monitor systematic up- or down-coding.

\section{Cream-skimming and dumping}

Service providers may decide to reorganise their services to include only simple, predictable cases that bring in a steady income (sometimes called cream-skimming), leaving patients with more complex needs (and higher financial risks) with less input (dumping).

\section{Combatting gaming}

Organisational and clinical entrepreneurs will use the $\mathrm{PbR}$ system to their advantage, so it is very important that there are clear measures of patient benefit and harm to make sure that patients do not lose out. Some of these measures need to be independent of the MHCT, since gaming of the tool for income necessarily would game the outcome derived from HoNOS. Up-coding on initial assessment both increases income and inflates future positive outcomes, so there is a double incentive to exaggerate cluster assignments. Although CROMs have value, it may be the PROMs and PREMs that are the most independent and transparent.

\section{Summary}

Mental health $\mathrm{PbR}$ and clustering constitute a highly disruptive financial mechanism for funding mental healthcare that brings the NHS closer to a market approach. There are still many challenges ahead and it is quite possible that mental health $\mathrm{PbR}$ may not work out. The purchaser/provider split is not a panacea for service improvement, and state-funded services can increase efficiency and quality without it (Timmins 2013).

Implementation has been put back more than once, but mental health PbR still appears to be on the way and services need to be prepared. Clustering will reduce clinical time for patients, but the impact can be lessened with good IT support and good training. Gaming will be easy and should be discouraged from the outset by emphasising probity and professionalism. Outcome measures will be introduced and these could help patients and practitioners understand what kind of care really works.

New providers, including big business and the voluntary sector, can enter the market. Small independent providers and individuals could benefit because they may be able to access money currently bound up in block contracts. There will also be losers in a market approach. Among service providers, the losers could be those that do not upgrade their IT systems and those that fail to engage and train their staff. Many clinicians just want to get on with their primary jobs. For them, the best approach to clustering is to try to do it accurately and quickly. The Care Pathways $\&$ Packages Project (CPPP) is developing the clustering currency. Updates on the progress of mental health $\mathrm{PbR}$ and clustering can be found on the CPPP website (www.cppconsortium. nhs.uk), along with their excellent web-based clustering algorithm.

\section{References}

Department of Health (2013a) Mental Health Clustering Booklet (Version 3.0) (2013/14). Department of Health (https://www.gov.uk/government/ publications/mental-health-payment-by-results-arrangementsfor-2013-14)
MCO answers

1 c $\quad 2$ e 3 b 4 a 5 b 
Department of Health (2013b) MHCT Algorithm Spreadsheet. Department of Health (https://www.gov.uk/government/publications/mental-healthpayment-by-results-arrangements-for-2013-14).

Department of Health (2013c) Mental Health Payment by Results Guidance for 2013-14. Department of Health (https://www.gov. uk/government/publications/mental-health-payment-by-resultsarrangements-for-2013-14)

Department of Health (2013d) Key Steps for Successful Implementation of Mental Health Payment by Results. Department of Health (https:// www.gov.uk/government/publications/mental-health-payment-byresults-arrangements-for-2013-14)

Jacobs R (2014) Payment by results for mental health services: economic considerations of case-mix funding. Advances in Psychiatric Treatment, 20: $155-64$.

Lee S, Forsyth K, Morely M, et al (2013) Mental health payment-byresults clusters and the Model of Human Occupation Screening Tool. OTJR: Occupation, Participation and Health, 33: 40-9.

Oyebode F (2007) Payment by volume (not results): Invited commentary on Payment by results in mental health. Advances in Psychiatric Treatment 13: 7-9.

Radhakrishnan V, Smith K, O'Hara J (2012) The Mental Health Clustering Tool for people with severe intellectual disability. Psychiatrist, 36: 454-8.
Royal College of Psychiatrists (2014) Royal College of Psychiatrists' Statement on Mental Health Payment Systems (formerly Payment by Results) (Position Statement PS01/2014). Royal College of Psychiatrists (http://www.rcpsych.ac.uk/pdf/PS01_2014x.pdf).

Smith A (1776) The Wealth of Nations. W Strahan \& T Cadell.

Tennant R, Hiller L, Fishwick R, et al (2007) The Warwick-Edinburgh Mental Well-being Scale (WEMWBS): development and UK validation. Health and Quality of Life Outcomes, 5: 63.

Timmins N, Ham C (2013) The Quest for Integrated Health and Social Care: A Case Study in Canterbury, New Zealand. The King's Fund.

Vize $\mathrm{R}$ (2013) An immensely delicate balance: the challenges for CCGs. BMJ, 346: 25.

Watts R, Donnelly L (2012) Don't leave patients in ambulances to hit A\&E targets, hospitals told. Daily Telegraph, 27 0ct.

Wing JK, Curtis RS, Beevor AS (1996) HoNOS: Health of the Nation Outcome Scales: Report on Research and Development July 1993December 1995. Royal College of Psychiatrists.

Wunderink L, Nieboer R, Wiersma D, et al (2013) Recovery in remitted first-episode psychosis at 7 years of follow-up of an early dose reduction/ discontinuation or maintenance treatment strategy: long-term follow-up of a 2-year randomized clinical trial. JAMA Psychiatry, 70: 913-20.

\section{MCOs}

Select the single best option for each question stem

\section{Clustering is:}

a a clinical process of allocating payments

b a financial process of allocating diagnosis

c a financial process using clinical terms

$\mathrm{d}$ a clinical process using financial terms

e an IT system combining diagnosis and payment.

\section{Clinicians:}

a need to cluster each patient only once

b should pick the cluster with the highest payment

c must recluster every 6 months

$\mathrm{d}$ can use diagnoses and clusters interchangeably

e can make the final clustering decision.

\section{Outcomes recommended by the} Department of Health in relation to mental health payment by results include:

\section{a CORE}

b SWEMWBS

c marital status

d physical health

e MOHOST.

\section{Down-coding:}

a reduces income

b increases income

c improves outcomes

$d$ is a common form of gaming

e cannot happen accidentally.
5 The Mental Health Clustering Tool:

a has no impact on clinical time with patients

$\mathrm{b}$ is a method of prospectively allocating payment for services

c uses only symptom scores

d cannot yet be supported by a computer algorithm

e does not address case mix. 